\title{
Axel Rover Paddle Wheel Design, Efficiency, and Sinkage on Deformable Terrain
}

\author{
Pablo Abad-Manterola, Joel W. Burdick, Issa A. D. Nesnas, Sandeep Chinchali, Christine Fuller, and \\ Xuecheng Zhou, California Institute of Technology and the Jet Propulsion Laboratory
}

\begin{abstract}
This paper presents the Axel robotic rover which has been designed to provide robust and flexible access to extreme extra-planetary terrains. Axel is a lightweight 2wheeled vehicle that can access steep slopes and negotiate relatively large obstacles due to its actively managed tether and novel wheel design. This paper reviews the Axel system and focuses on its novel paddle wheel characteristics. We show that the paddle design has superior rock climbing ability. We also adapt basic terramechanics principles to estimate the sinkage of paddle wheels on loose sand. Experimental comparisons between the transport efficiency of mountain bike wheels and paddle wheels are summarized. Finally, we present an unfolding wheel prototype which allows Axel to be compacted for efficient transport.
\end{abstract}

\section{Motivation}

$\mathrm{D}$ ESPITE the great successes of the Mars Exploration Rovers [1], some of the richest potential science targets for future exploration missions lie in terrains that are inaccessible to state-of-the-art Martian rovers, thereby limiting our ability to carry out in situ analysis of these rich opportunities. For example, bright new deposits, which may be ice flows, have been discovered hundreds of meters below the rims of steep craters in the Centauri Montes regions on Mars (Figure 1).

While the Opportunity rover has imaged layers of bedrock in the vertical promontories of Cape St. Vincent in Victoria crater, these geological features are currently inaccessible to conventional sampling methods. High-resolution orbiter images of stratified deposits of ice and dust reveal a very challenging terrain, which if it could be navigated, would provide important clues to the geological and hydrological past of Mars [2]. The recently reported Martian methane plumes [3] rise over heavily cratered terrains in the Arabia Terra and Syrtis Major regions. Without new mobility platforms, it will be difficult to access the surface of this

The work of this collaboration between Caltech and the Jet Propulsion Laboratory was performed at JPL under contract to the National Aeronautics and Space Administration. The authors sincerely appreciate the sponsorship of the Exploration Systems Mission Directorate and Solar Systems Exploration program: Dr. C. Moore, Dr. S. Khanna, Dr. T.Y. Yan, Dr. J. Cutts, and Dr. K. Reh. We also appreciate the support of Dr. S. Hayati, Dr. R. Volpe and Dr. G. Udomkesmalee of JPL.

Pablo Abad-Manterola is with the California Institute of Technology, Pasadena, CA 91125 USA (phone: 847-867-1855; e-mail: pablo@caltech.edu).

Joel W. Burdick, is with the California Institute of Technology, Pasadena, CA 91125 USA (phone: 626-395-4139; e-mail: jwb@robotics.caltech.edu).

Issa A.D. Nesnas is with the Jet Propulsion Laboratory, Pasadena, CA 91109 USA (phone: 626-318-9002; e-mail: nesnas@jpl.nasa.gov). region to assess if the methane has a biological or geological origin. Similarly, Titan, Europa, Enceladus, and the Earth's moon also offer challenging surface features with associated scientific targets. A new generation of planetary exploration robots is needed to access the challenging terrains in order to probe, sample and measure. New inquiries of this sort could result in significant scientific rewards.

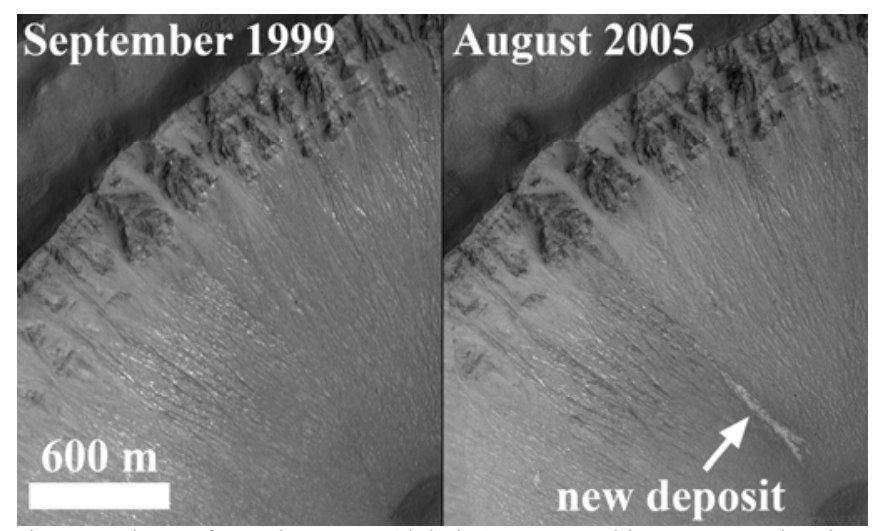

Fig. 1. Photos from the Mars Global Surveyor orbiter camera showing recent flows in a crater of the Centauri Montes region.

\section{ROBOTIC MOBILITY FOR EXTREME TERRAIN}

Mechanisms and algorithms for robotic mobility in steep and complex terrains have been investigated for several decades. Proposed approaches include multi-legged quasistatic walkers [4], bipedal walkers [5], hopping machines [6], snake-like mechanisms [7], and wheeled vehicles with complex wheel designs [8] or articulated suspensions [9]. Critical issues in evaluating the viability of these methods for space applications include the ability to navigate rough terrain, the approach's inherent robustness and mechanical complexity, the total system mass and volume, the energy required per traverse distance, the ability to carry out in situ scientific studies and sample gathering, and the ability to recover from faults. Most previously proposed methods have one or more drawbacks with respect to these criteria.

For exploring challenging topographies, an actively controlled tether combined with a conventional mobility platform (using e.g. wheels, legs, or tracks) may provide a useful means to enable very steep terrain access. One such example was the Dante tethered robot [10] that descended into the Mt. Spurr volcano in 1994 using its tether and an 8legged walking frame. In the 1990's, following orbital imagery of Mars stratigraphy, a number of different mission 
concepts were proposed for in situ science investigations that included legged and wheeled robots. Cliff-bot, a wheeled robot that used a dual tether system to help manage its traverse across a cliff face, has been demonstrated on cliff faces in Svalbard, Norway [11]. In addition to the legged and the multiple wheeled robot approaches, some of the earlier concepts also advocated the potential advantages of using lightweight tethered platforms, though none of these efforts led to an implementation.

\section{THE AXEL System OVERVIEW}

In order to provide access and in situ sampling in areas of extreme terrain, the Jet Propulsion Laboratory (JPL) and the California Institute of Technology (Caltech) have been collaborating to develop the Axel rover. Axel is a minimalistic robot consisting of two wheels connected by a central cylindrical body, a caster arm, and an actively controlled tether passing through the caster arm (Figure 2). The caster arm, in addition to controlling the tether, also provides a reaction force against the terrain necessary to generate forward motion when travelling on flat ground.

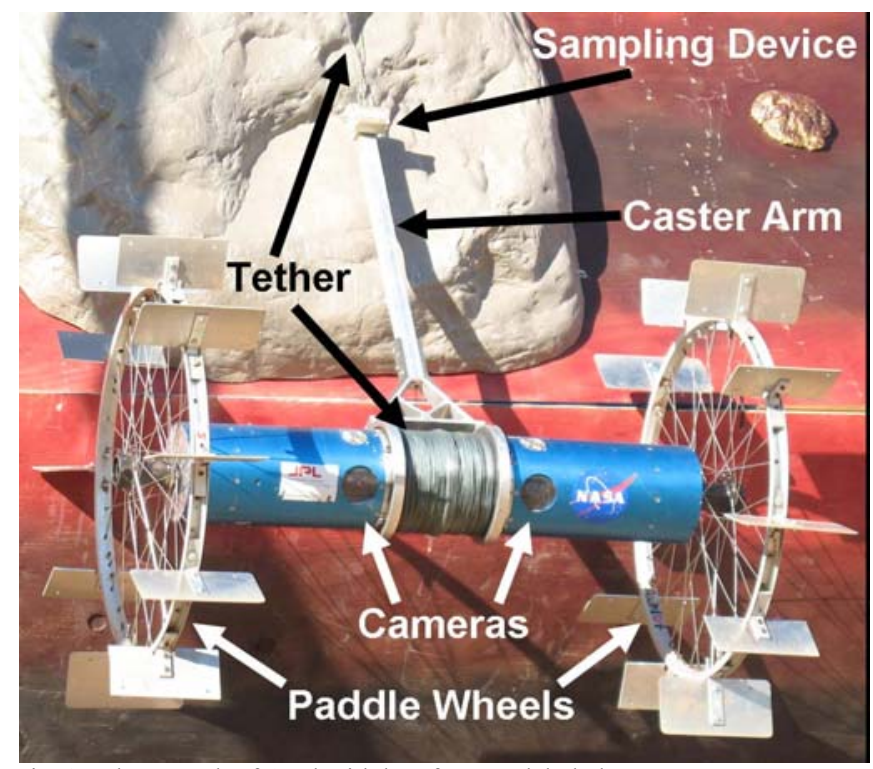

Fig. 2. Photograph of Axel with key features labeled.

Axel's minimalist design overcomes some of the limitations found in prior tethered robots. Dante's operation on Mt. Spurr was cut short when it tipped over-it had no built-in mechanism to recover an upright posture. Due to its symmetry, Axel has no upside-down posture and thus does not suffer from this failure mode. Future wheel designs will be hemispherical, ensuring that Axel cannot rest stably on its side. Cliff-bot similarly has no tip-over recovery and uses two tethers. Like Dante, Axel's tether is paid out by an onboard motor, an advantage compared to Cliff-bot's more complicated off-board tether management system. Due to Axel's low mass, on-board battery, and wireless communication link, the tether is a simple high strength cord as opposed to Dante's heavy tether with embedded power and signal conductors.

Axel's minimalist design satisfies many of the severe constraints imposed by space mission design. Because the rover uses only three actuators to control its wheels, caster arm, and tether, its total mass is low (the current prototype weighs $\sim 22 \mathrm{~kg}$ ). Its simplistic design improves mechanical robustness. All of its electronic components (motors, sensors, and computers) can be centralized in the body, simplifying thermal control design for operation in extreme cold.

\section{A. Mission Concept}

We expect Axel to operate in hazardous terrain via the use of a host platform as an anchor. Since Axel's body acts as a winch, the host platform requirements are reduced to a simple mount. The host platform could be a lander, a larger rover, a habitat, or even an astronaut. Once the anchor point is secured, Axel can descend over steep promontories, navigate through rocky terrain, take images, collect soil samples, and then return by reeling in its tether. Figure 3 portrays a hypothetical scenario in which Axel is deployed from the Mars Science Laboratory (MSL) [12], an example of a host platform that could potentially carry one or more Axels as a method of sampling in extreme terrain.

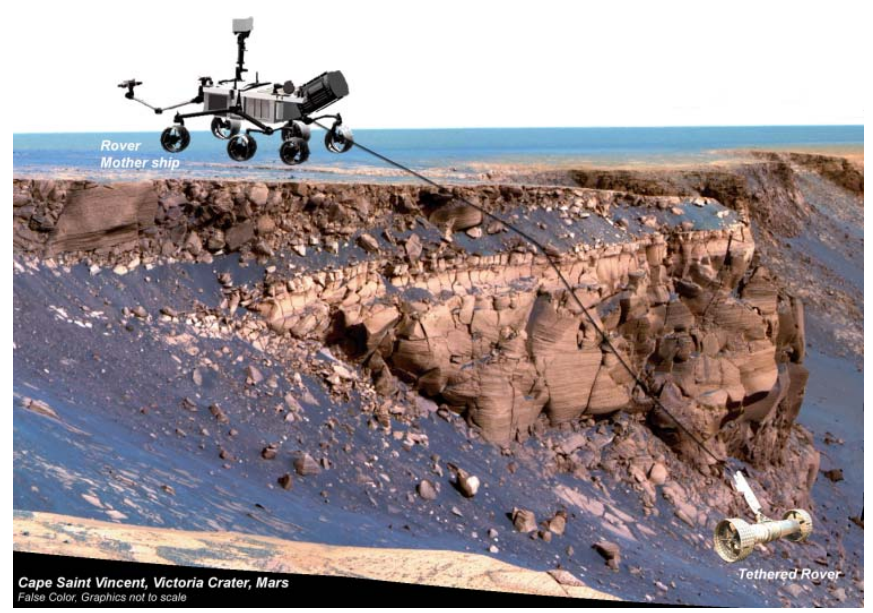

Fig. 3. Proposed mission concept overlaid on false color image of Victoria Crater. Note that the rover graphics are not to scale.

There are some key advantages to this tethered approach for planetary exploration missions. The risk to overall mission success of descending into craters or similar topographies is minimized, as the host can detach the tether should Axel fail and then continue with other mission objectives. Axel is also small and light enough for more than one copy to be hosted from an MSL class rover. Because Axel can operate without a tether, depending on the nature of the failure, Axel may be able to continue its exploration to some level. Second, since Axel itself is the winch, the tether is laid over the terrain as the rover descends, and it is collected as the rover returns to the host. In contrast to a winch mounted on the host, our approach minimizes abrasion on the tether from rocks and cliff faces. Finally, the anchor and tether system allows Axel to travel over cliff 
promontories with slope angles greater than $90^{\circ}$, which would not be possible with an independent wheeled rover.

\section{B. Prototype System}

The current Axel prototype is shown in Figure 2. It measures $1 \mathrm{~m}$ from wheel edge to wheel edge and $0.75 \mathrm{~m}$ from the body's center to the end of the caster arm. The paddle wheels are $0.74 \mathrm{~m}$ in diameter.

Axel's body houses and protects all of its hardware and electronic components. Computations are performed by a $700 \mathrm{MHz}$ Pentium processor with $128 \mathrm{MB}$ of RAM and a 2 GB solid state drive. The wheels are each driven by a $153 \mathrm{~W}$ Servodisc drive coupled to a harmonic drive. The entire system is controlled remotely via an 802.11 b wireless link. Axel's 24V, 4.2 amp-hour battery allows for one hour of continuous driving before it must be recharged. A safety circuit, which brakes the caster motor in case of sudden power loss, ensures a slow and controlled descent, eliminating the need for a safety tether.

Stereo cameras are located as shown in Figure 2 in order to make room for the tether. The two PointGrey $1024 \times 768$ color Firewire cameras have a $25 \mathrm{~cm}$ baseline. Fujinon lenses with $105^{\circ}$ field-of-view provide reasonable visibility so that a human teleoperator can drive the rover from a remote position. In tumbling mode, primarily used to reel and unreel the tether during ascent/descent, Axel's cameras rotate with its body, producing images at different pitch angles. To control the pitch at which images are acquired, an inertial measurement unit (MicroStrain 3DM-GX1) triggers image acquisition. By acquiring consecutive images at the same pitch, a more intuitive image map can be constructed, which simplifies human teleoperation as well as autonomous map-based motion planning.

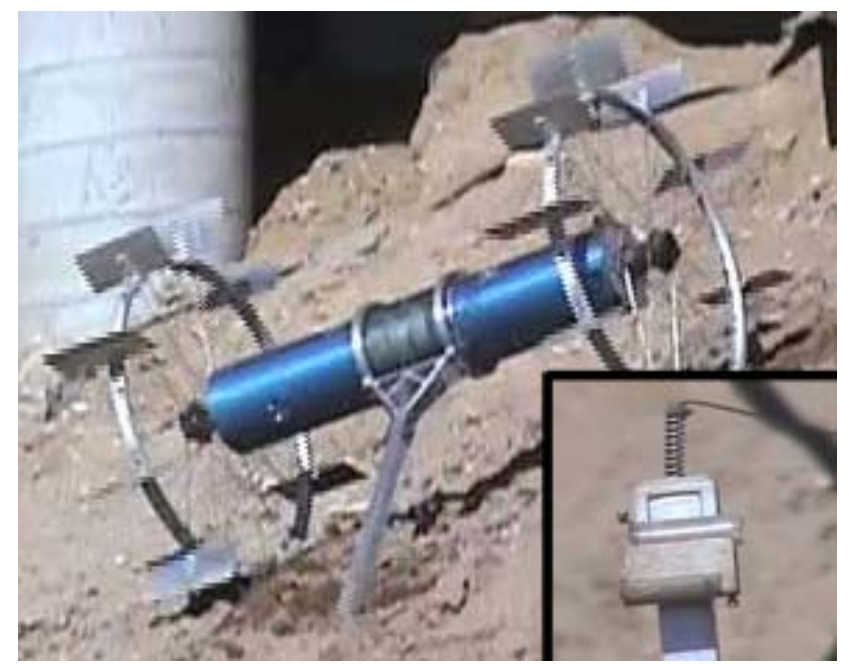

Fig. 4. Axel taking a sample by pointing the caster arm into the ground and turning in place. Sand enters through the openings on the ends of the removable sampling tubes (inset).

At present, we have a simple soil sampling system to allow for experimentation with sample acquisition strategies in extreme terrain. This sampling device (Figure 4 inset) features two sample containers mounted on the end of the caster arm, perpendicular to its long axis. After pushing the caster arm into the ground, Axel oscillates around its center, thereby scooping soil into the tubular containers. The tubes face opposite directions, enabling the collection of two separate samples. Furthermore, the containers are detachable so that the host platform rover could potentially remove the sample for scientific analysis. This method works well for loose soil and on slopes ranging from 0 to 40 degrees.

A summary video of Axel descending down a 90 degree slope, collecting a soil sample, traversing obstacles, and ascending back up to the host platform is included as an accompaniment to this paper. It can also be found at:

http://robotics.caltech.edu/ pablo/axel/movies_2009.01.

Because Axel's extreme terrain mobility is heavily dependent upon the performance of its wheels, the following sections explore a novel wheel design and its capabilities and characteristics on deformable terrain.

\section{WheEl Performance OVER Obstacles}

Descending crater walls and navigating over loose sediment and rocky terrain persists as a difficult challenge for rovers, whose performance in these scenarios depends greatly on the type of wheels they carry. Thus, we have exerted some effort on the design and optimization of Axel's wheels for our particular goal of extreme terrain exploration. As seen in Figure 2, Axel uses an unusual wheel design that combines a conventional wheel rim with evenly spaced "paddles," similar in concept to the Whegs Robots [13]. These wheels provide a motion that is roughly a hybrid of rolling and walking. A short summary of the comparison between paddle wheels and conventional wheels over obstacles will now be presented. For a more complete analysis, refer to our previous publication on wheel design [14].

For a standard, circular wheel to achieve a positive acceleration over an obstacle, two conditions must be met:

$$
\tau>m g r \sin \theta ; \mu>\tan \theta
$$

where $\tau$ represents the torque applied to the wheel by the motor, $m$ is the mass of the wheel, $g$ is the gravitational constant, $r$ is the radius of the wheel, $\mu$ is the coefficient of friction between the wheel and the obstacle, and $\theta$ is the angle between the vertical and the point of contact of the obstacle along the rim of the wheel.

Without the aid of a tether, the wheel cannot surpass obstacles whose contact point height is greater than one wheel radius above the ground plane. However, when outfitted with paddles, Axel's wheels are able to surpass much larger obstacles than their smooth, circular counterparts. In the simple 2-dimensional model, the necessary conditions for a paddle wheel to traverse over an obstacle without slipping are:

$$
\tau>m g(r+l) \sin \theta
$$




$$
\mu>\frac{g\left(I+m(r+l)^{2}\right) \cos \theta}{\lg \sin \theta+\tau(r+l)} .
$$

In these equations, we have added the parameters $l$, which denotes the length of the paddle, and $I$, which is the rotational inertia of the wheel. Note that since the lower bound on the coefficient of friction is inversely proportional to input torque, this bound can be reduced by increasing the wheel torque. Thus, with a paddle wheel design, for a small increase in the required torque, it becomes easier to travel over obstacles without slipping, especially as the contact point angle increases.

In summary, a simplified physics model of the paddle wheel predicts that it will perform better than a traditional wheel at higher contact point angles, which agrees with experiments conducted in the Mini Mars Yard at JPL. However, while the paddle wheels have natural advantages compared to conventional wheels when it comes to maneuvering over rocks, such wheels may have disadvantages as well. The next two sections explore the extent to which paddle wheels may sink in soft planetary soil and the penalty in efficiency of a paddle wheel.

\section{SinKage Model}

A paddle wheel could potentially sink deeply into soft soils as may be found on the Earth's moon or on Mars. Moreover, the ability to develop control schemes and motion planning algorithms for Axel depends greatly on how we model the wheel/soil interaction. As a starting point for more advanced traction models, an accurate calculation of soil penetration by a paddle is a necessary step. This section develops a preliminary quasi-static model to estimate the depth of paddle wheel sinkage in loose sand based on Bekker's equations for deformable terrain. We then compare the model to sinkage data taken in the Mini Mars Yard at JPL.

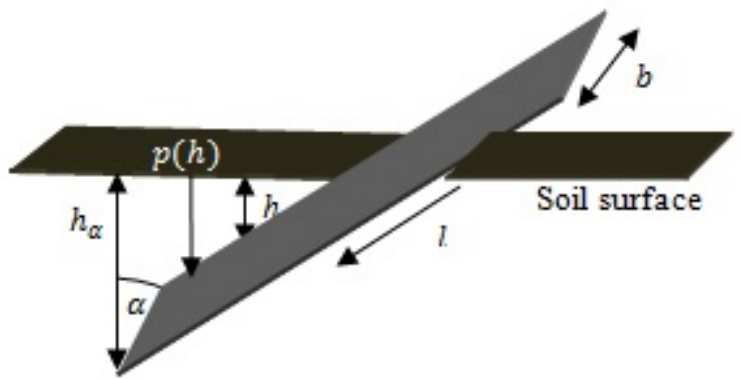

Fig. 5. Submerged flat plate at arbitrary angle $\alpha$ with key parameters labeled. The plate models a paddle.

Bekker's pressure-sinkage equation (which relates the depth to which a thin vertical plate penetrates the soil to soil characteristics and the pressure on the plate) is

$$
p(h)=\left(\frac{k_{c}}{b}+k_{\phi}\right) h^{n}
$$

where $h$ is the depth of the blade penetration into the ground, $b$ is the width of the plate, $p$ is the pressure on the plate, and $k_{c}, k_{\varphi}$, and $n$ are constants based on the soil type (dry sand in our calculations and experiments) [15]. We incorporate this equation into our sinkage model by considering Axel's paddles as submerged plates at some arbitrary angle, as in Figure 5.
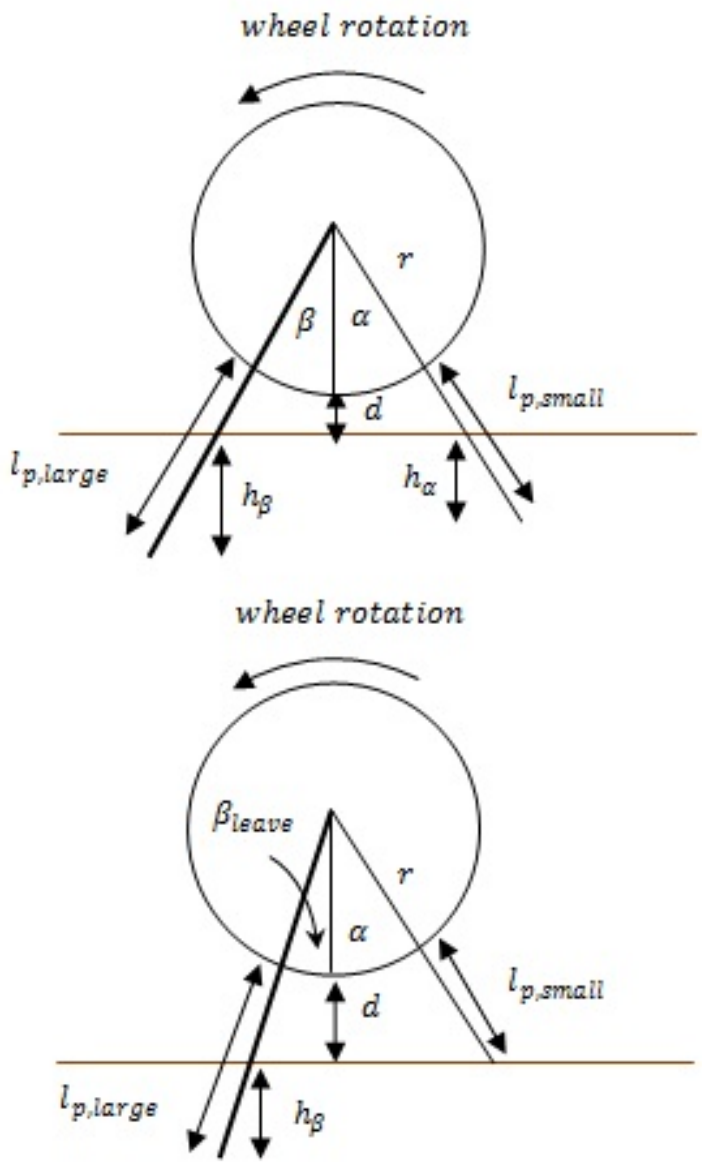

Fig. 6. Above: paddle wheel with 2 paddles submerged in the ground. Below: $\beta_{\text {leave }}$ is the angle at which one paddle just loses contact with the ground.

Integrating the pressure equation along the direction $l$ and approximating $n=1.1 \approx 1$ in order to realize an analytic expression, we find the total vertical force on one paddle to be approximately

$$
F_{\text {paddle }}=\frac{b}{2 \cos \alpha}\left(\frac{k_{c}}{b}+k_{\phi}\right)(h)^{2} .
$$

Axel's wheels feature two different types of paddles, one larger and one smaller, mounted in an alternating fashion along its rim. Furthermore, while the paddle wheels rotate, Axel switches between having one and two paddles in contact with the ground. Thus, in determining the total vertical force from the ground to the wheels, it is necessary to determine $\beta_{\text {leave }}$, the angle at which one paddle loses 
contact with the ground as the wheel drives forward. As can be seen from the diagram in Figure 6, this angle is calculated as

$$
\beta_{\text {leave }}=\alpha-\theta_{\text {sep }}=\cos ^{-1}\left(\frac{r+d}{r+l_{p, \text { small }}}\right)-\theta_{\text {sep }}
$$

where $\theta_{\text {sep }}$ is defined as the separation angle between the paddles, measured as 36.8 degrees. Additionally, the force on each paddle can be calculated as a function of the single variable $d$, the distance from the bottom of the wheel rim to the ground. In static equilibrium, the force from the paddles will balance with the weight of the rover.

Two sets of measurements of the wheel height, $d$, were carried out in the JPL Mini Mars Yard for various values of $\beta$, the angle between the larger paddle and the vertical. The rover was initially placed on a flat patch of sand with both paddles at approximately equal angles to the vertical. The wheel motor was then driven to rotate the wheel, and measurements of $d$ were taken until the paddle passed the vertical and the rover tipped to the other side. The data is plotted alongside the theoretical model in Figure 7, where the wheel height is plotted vs. the large paddle angle from the vertical. The data from the two tests fits the model reasonably well and deviates mostly in the extremes where the large paddle is almost vertical or close to leaving the ground. It may be necessary to develop a different model for these scenarios in order to more closely approximate the observed results.

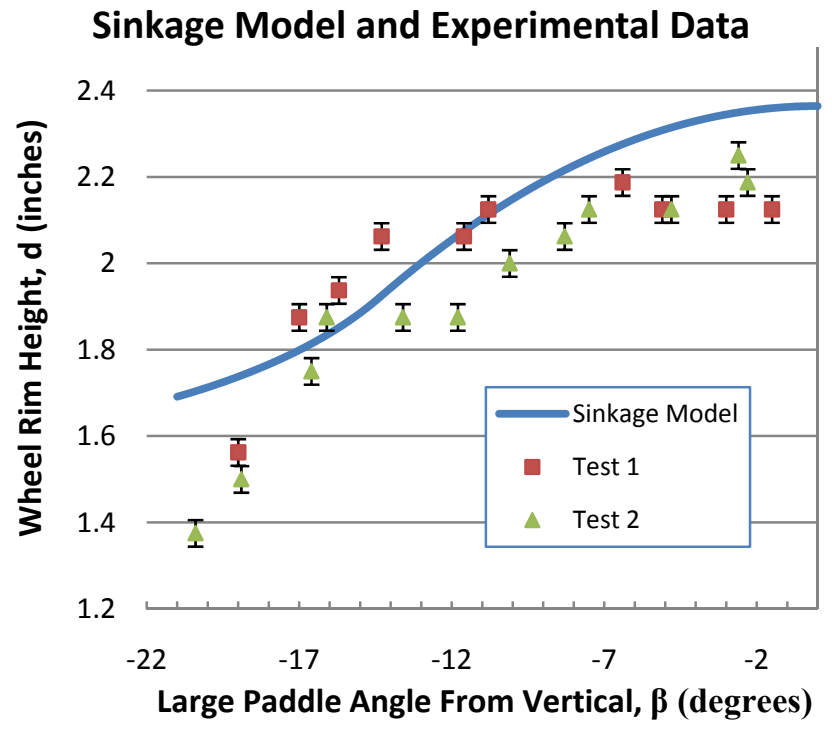

Fig. 7. Experimental sinkage data overlaid with the theoretical model.

The model and our experiments suggest the benefit in rock climbing ability gained by the introduction of the paddle does not lead to a wheel design which might sink deeply into soft soils and therefore become bogged down. Next we consider the impact of the paddle wheel design on traverse efficiency.

\section{WHEEL EFFICIENCY}

In section IV we compared the performance of traditional wheels and paddle wheels over obstacles. While the ability to surpass large rocks is an important characteristic of any wheel design for rovers on extreme terrain, efficiency is also paramount for robotic planetary explorers with severely constrained power sources. Craters, for example, may contain areas which are shrouded in permanent shadow, limiting the amount of solar energy that can be collected. Furthermore, because of the difficulty and cost associated with pinpoint landing on the moon or Mars, the landing zone of Axel's host platform may be extremely wide. As a result, an Axel rover may need to initially travel up to $2 \mathrm{~km}$ over flat terrain in order to reach the edge of the crater it will explore. Thus, efficiency of the wheel is a key concern.

The development of an analytical model that can accurately predict the efficiency of a paddle wheel is an ongoing challenge. In this paper, we take a more empirical approach, comparing the measured energy expenditure of the paddle wheels and a pair of mountain bike tires (with essentially the same diameter) while both types of wheels travel over flat ground consisting of loose playground sand. One can intuitively hypothesize that the jerky, hybrid rolling/walking motion of the paddle wheel would be much less efficient than that of a smooth bike tire over flat ground. Experiments conducted with Axel in the Mini Mars Yard at JPL quantify efficiencies and validate this hypothesis.

\section{Power vs Time at 6.5 RPM}

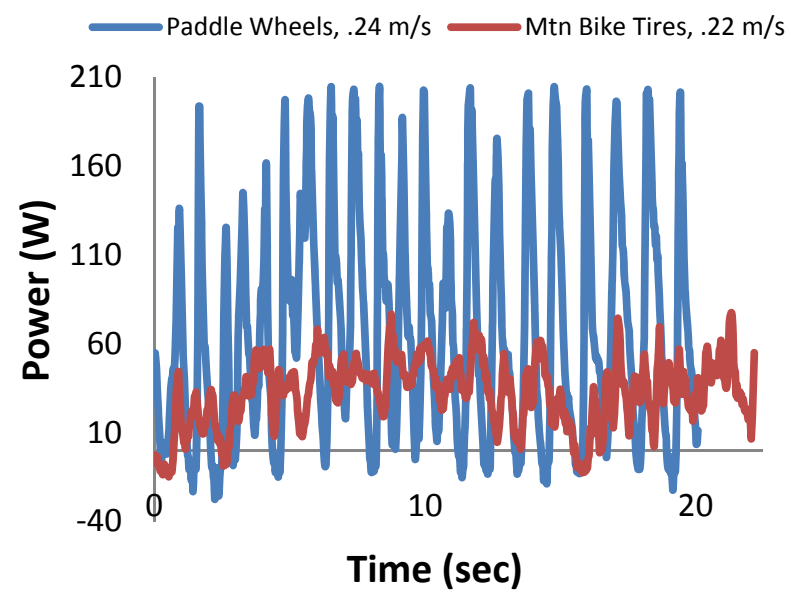

Fig. 8. Power vs time over a 16 foot straight line course for both the paddle wheels and mountain bike tires. The caster arm drives the body and wheels at an angular rate of $6.5 \mathrm{rpm}$.

We delineated a straight, $16 \mathrm{ft}$. long course to drive along at different speeds with each wheel type. Three trials were conducted for each speed with a total of five different speeds, resulting in 15 trials for each wheel type. During each trial, Axel was driven by pushing the caster arm into 
the ground, causing the body and wheels to rotate at the same angular rate. Voltage and current readings for the caster were recorded from the Elmo Whistle motor controller. From these measurements one can approximate the amount of power and energy required to complete the traversal of the test course.

A plot of power versus time is shown in Figure 8. Notice the large amplitude, sinusoidal nature of the power curve for the paddle wheels. This corresponds to the increased torque required by the motor to push the wheel to the edge of the paddle and then tumbling forward once the paddle has passed the vertical. The mountain bike tire, on the other hand, is relatively smooth and consistent in terms of its power requirements over flat ground. The difference in linear velocity between the two wheels is a result of the fact that the paddle wheels have a slightly larger effective radius.

For each speed, the power requirements were averaged over the three trials and divided by the velocity in order to calculate the energy required by the rover to travel one meter. The results of this calculation as a function of linear velocity can be seen in Figure 9. At any given rover speed, the energy required by the rover is approximately doubled when using the paddle wheels, which supports our initial hypothesis. Furthermore, energy requirements increase dramatically at the lower speeds.

\section{Energy Consumption vs Speed}

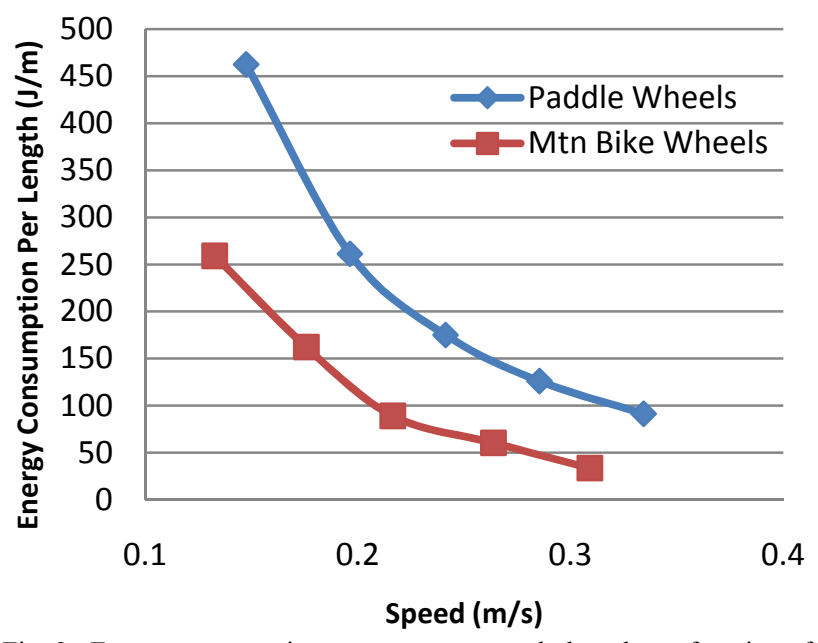

Fig. 9. Energy consumption per meter traversed plotted as a function of the rover speed.

Thus, not only are the paddle wheels less efficient while travelling on flat ground, but, as can be seen in Figure 8, the non-smooth motion of the rolling paddle wheel can cause the instantaneous power demand to be approximately 5 times greater than that of the standard wheel. Nevertheless, the increased performance over obstacles and steep slopes prevents us from abandoning the paddle wheel concept altogether. Axel is primarily designed to be capable of navigating extreme terrain. Losses in efficiencies in certain environments can be mitigated by the existence of a reliable power source, such as the mother rover, which could transmit energy through the tether. The mother rover could also serve as reliable transport vehicle, efficiently carrying Axel over large distances to the edge of craters, where the smaller rover is ideal for exploration. However, improving the efficiency of the paddle-wheel concept is clearly a high priority.

\section{COllapsing WheEl Design}

Simple calculations, as well as practical intuition, suggest that increasing the radius of a rover's wheels improves its ability to traverse over larger obstacles. Nevertheless, the cost of a payload launch to a distant planet or moon rises exponentially with volume of the payload. Furthermore, Axel is designed to be mounted as part of a larger, marsupial rover, which has a limited carrying capacity in mass and volume. While the fixed paddle-wheel design offers many advantages on extreme terrains, it is highly inefficient in terms of launch volume.

In order to retain the mobility advantages of the paddle design, while also paying heed to the severe constraints of launch volume, we have developed an expandable wheel prototype which is designed to collapse during launch and unfold to a large diameter when the rover is first deployed.

Dynamic unfolding, the ability to expand and collapse repeatedly, would require one additional motor for each wheel. Thus, in keeping with the philosophy of minimizing complexity, we have opted for a design which only unfolds once upon deployment from the mother rover. The wheel was also designed to weigh not more than $2.5 \mathrm{~kg}$ and protect the body from sharp rocks without blocking the caster arm's movements.

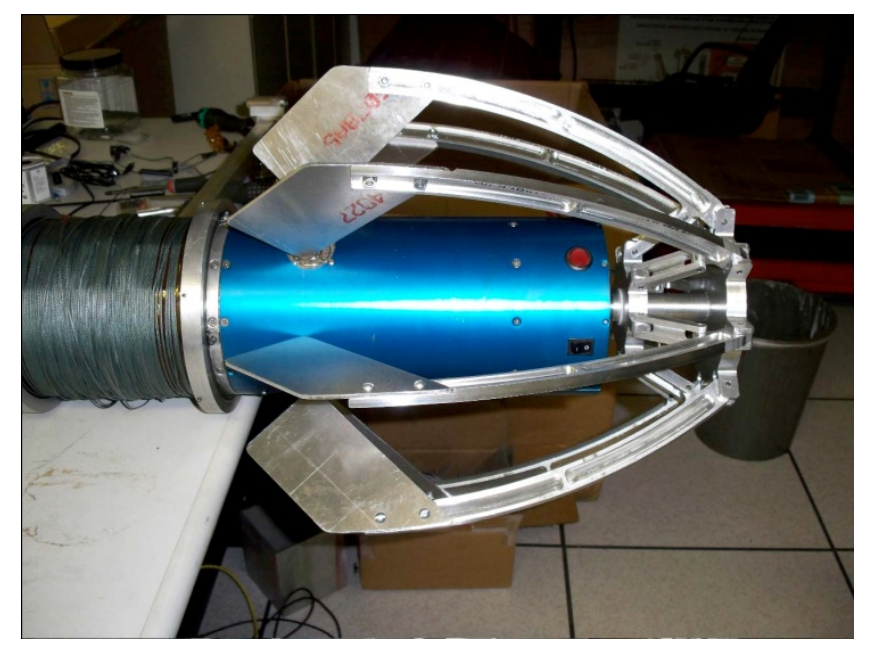

Fig. 10. Unfolding wheel prototype collapsed around Axel. Secondary plate can be seen just adjacent to the rover's body.

The prototype was constructed from aluminum and roughly models the concept of an unfolding umbrella. The wheel is expanded when a secondary plate is pushed along the shaft (Figure 10). It has a collapsed diameter of 16 in. and expands to 29 in. when completely unfolded. The spokes (or "arms") arch towards the center of body, protecting the electronic components and scientific measurement tools 
from rocks and other terrain features. The rounded shape of the wheel spokes is designed to help the rover self-right should it ever fall or tip over on its end.

While only one wheel has been constructed to date, it was still possible to test its performance in the Mini Mars Yard at JPL. The wheel performed well travelling over loose playground sand and rocks approximately $1 / 3$ of the wheel diameter (Figure 11). Mounted alongside the previous paddle wheel design, the unfolding wheel successfully climbed a one-foot step obstacle.

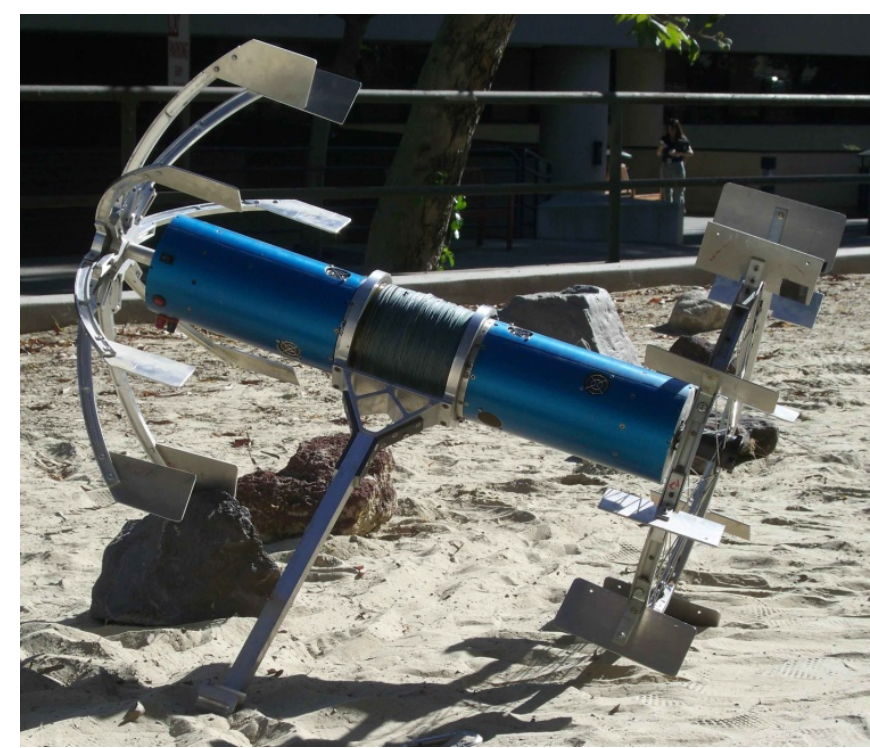

Fig. 11. Unfolding wheel prototype in expanded state mounted alongside original paddle wheel. The wheel is travelling over a rock approximately $1 / 3$ of its diameter.

In summary, the unfolding wheel design holds much potential for combining a small-volume deployment with a large-radius wheel capable of traversing many obstacles over extreme terrain. Conceivably, one could also use an inflatable wheel for such a purpose. However, inflatable wheels do not have the stiffness needed for rock traversal, and they are very likely to be punctured in the extreme terrains in which we envision operating Axel.

\section{CONCLUSIONS AND FUtURE WORK}

We presented a minimalist tethered robotic rover, Axel, whose low mass, simplicity, and robustness make it a viable candidate for future explorations of extreme planetary terrains. While we envision that Axel will be deployed via a tether from a host rover or lander, its simplicity and low mass would allow it to be used in various mission designs.

Axel realizes excellent mobility by the cooperative effort of its actively managed tether and its novel wheels. This paper focused on issues of wheel design, which is one of the essential components for successful deployment of Axel in extreme terrain. We have shown that a modified wheel design provides Axel with a greater capability to traverse rocky terrain and climb moderate slopes without a tether. We have also demonstrated the effectiveness of the paddle wheel design in travelling over large obstacles. We also summarized experimental work showing Axel's ability to negotiate simulated extreme terrains of the type that might be encountered in future missions. Ongoing and future work seeks to develop a more sophisticated computational model involving the complex wheel-soil interaction. Such improved models would then lead to advanced planning algorithms that would allow Axel to complete its goals autonomously.

A future version of Axel will feature additional scientific measuring tools and a new tether capable of transmitting power and communications. The new rover will take measurements at steep slopes along crater walls and it will have a maximum traverse distance of at least $1 \mathrm{~km}$.

\section{REFERENCES}

[1] http://marsrovers.jpl.nasa.gov/home/index.html

[2] E. Kraal, M. Dijk, G. Postma, M.G. Kleinhans, "Martian stepped-delta formation by rapid water release," Nature 451, Feb. 21, 2008, pp. 973976.

[3] http://www.nasa.gov/mission_pages/mars/news/marsmethane.html

[4] K.J. Waldron, Machines that Walk: the Adaptive Suspension Vehicle, MIT Press, Cambridge, Mass., 1989.

[5] M. Hirose and K. Ogawa, "Honda humanoid robots development," Phil. Trans. Royal Soc. A, 365(1850):11-19, Jan. 2007.

[6] J. Burdick an P. Fiorini, "Minimalist Jumping Robots for Celestial Exploration," Int. J. Robotics Research, vol. 22, no. 7-8, pp. 653-674, Aug. 2003.

[7] G.S. Chirikjian and J.W. Burdick, "The Kinematics of HyperRedundant Locomotion," IEEE Trans. Robotics and Automation, vol. 11, no.6, pp. 781-793, Dec. 1995.

[8] M. Lamboley, C. Proy, L. Rastel, T.N. Trong, A. Zashchiriski, and S. Buslaiev, "Marsokhod Autonomous navigation tests on a Mars-like Terrain," Autonomous Robots, 2(4):1573-7527, Dec. 1995.

[9] D. Miller, T.L. Lee, "High-Speed Traversal of Rough Terrain Using a Rocker-Bogie Mobility System," Roving over Mars, Mechanical Engineering, April 1998, pp. 74-77.

[10] J. Bares and D. Wettergreen, "Dante II: Technical Description, Results and Lessons Learned," Int. J. Robotics Research, Vol. 18, No. 7, July, 1999, pp. 621-649.

[11] E. Mumm, S. Farritor, P. Pirjanian, C. Leger, P. and Schenker, "Planetary cliff descent using cooperative robots," Autonomous Robots, Vol. 16, No. 3, May, 2004, pp. 259-272.

[12] http://mars.jpl.nasa.gov/msl/

[13] http://biorobots.cwru.edu/projects/whegs/

[14] P. Abad-Manterola, J.W. Burdick, I. Nesnas, J. Cecava, "Wheel Design and Tension Analysis for the Tethered Axel Rover on Extreme Terrain," IEEE Aerospace Conference, 7-14 March 2009.

[15] M.G. Bekker, Off-The-Road Locomotion, University of Michigan Press, Ann Arbor, Mich., 1960. 